\title{
Mathematic simulation of the power interchange in the interbucket space of coaxially located impellers of the centrifugal turbo machine
}

\author{
Sergey Podbolotov ${ }^{1, *}$, Anatoly Kolga ${ }^{1}$, and Natalja Dyorina ${ }^{1}$ \\ ${ }^{1}$ Nosov Magnitogorsk State Technical University, Magnitogorsk, Russia
}

\begin{abstract}
The article presents the results of mathematic simulation to determine the effect of geometric parameters, coaxially installed impellers, a centrifugal turbo machine on the developed pressure, depending on the mode of operation (frequency and relative direction of the impellers rotation). It is established that irrespective of the operation mode, the influence of the circumferential velocity, the ratio of the input and output diameters and the width of the impeller on the value of the pressure developed by the turbo machine is invariable. While the influence of the density of the blades system decreases with the counter rotation of the impellers.
\end{abstract}

\section{Introduction}

The complexity of the turbo machine geometry and the presence of moving elements inevitably lead to the need for segmenting the water-conveyance system. This is mainly due to the presence of stationary flow ranges (a spiral chamber, a cascade of stator columns with blades of the guide device, a suction pipe) in the turbo machine and the interloper channels rotating together with the impeller. In addition, segmentation is often caused by the requirement to build quality rows (close to orthogonal, condensing to walls and flow features). Finally, segmentation is necessary to overcome the constraints on the topology of the computational domain imposed by the numerical method of solving the equations of motion $[1,2]$.

The flow simulation in a turbo machine can be carried out in various approximations. One of the most common and effective approaches is a stationary cyclic formulation, in which it is assumed that the flows along all the interloper channels of the impeller are the same. In this case, the calculation is implemented only in one of the channels of the apparatus and the wheel, and on the lateral boundaries of the channels the flow periodicity conditions are set. In order to transfer flow parameters from rotating segments to stationary segments and vice versa, their values are averaged in the circumferential direction $[3,4]$.

\footnotetext{
*Corresponding author: Podbolotov_Sergey@mail.ru
} 


\section{Methodology}

In order to simplify the understanding of internal processes and reduce the number of members of the mathematical apparatus, the basis of these studies is a two-stage model of a centrifugal turbo machine [5]. The influence of the outer stage on the inner stage is excluded, thus the process of power interchange on the blades of its impellers is not considered [6].

The power interchange between the blades of the turbo machine and the flow of fluid is characterized by the value of the theoretical pressure (head). The value of the latter is found from the well-known Euler equation:

$$
\mathrm{H}=\frac{1}{\mathrm{~g}}\left(\mathrm{u}_{2} \mathrm{c}_{2 \mathrm{u}}-\mathrm{u}_{1} \mathrm{c}_{1 \mathrm{u}}\right)
$$

where $\mathrm{u}$ - tip speed;

$\mathrm{c}_{\mathrm{u}}$ - whirl velocity.

The operation analysis of a centrifugal turbo machine with a coaxial arrangement of impellers is made on the basis of the construction of velocities parallelograms. The kinematics of the flow on the blades of the outer impeller is studied under different operating conditions. Changing the operating modes is made by varying the flow rate.

The smooth inlet of the flow into the intervane channel of the impeller is provided only in the design mode of operation $[7,8]$, when the feed rate is $Q_{T}=Q_{T O}$, in accordance with this mode, the input angle of the blade $\beta_{1}$ is selected. The vector of the mean absolute velocity of flow $c_{1}$ decomposes into two components: the transported $u_{1}$ and $\omega_{1}$, moreover the direction of the latter coincides with the tangent to the axis of the intervane channel.

If the turbo machine feeds with deviation from its calculated value, the mean relative velocity vector $\bar{\omega}_{1}$ deviates from the tangent direction to the axis of the intervane channel and can be decomposed into two components, one of which $\omega_{1}^{\prime}$ is axial which coincides with the tangent to the axis of the intervane channel, and the second $\omega_{1}^{/ /}$is a circumferential, is directed parallel to the vector $\bar{u}_{1}$. Moreover, in the $Q_{T}<Q_{T O}$ mode, the vector $\omega_{1}^{/ /}$is directed opposite to the vector $\bar{u}_{1}$, and for $Q_{T}>Q_{T O}$ coincides with it. The corresponding parallelograms of the velocities at the entrance to the row of the blades in relation to the general case of supplying the flow to the impeller are shown in Fig. 1. Here, the velocity vectors are denoted by the indices 11 for $Q_{T}<Q_{T O}, 10$ for $Q_{T}=Q_{T O}$ and 12 $Q_{T}>Q_{T O}$.

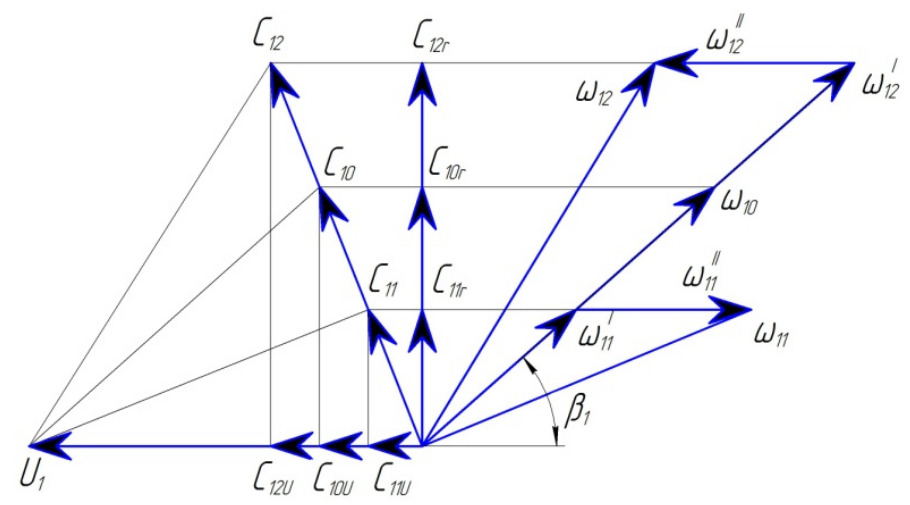

Fig. 1. Parallelograms of the average flow velocities at the inlet to the impeller of a centrifugal turbo machine 
The circumferential component of the relative flow velocity of the fluid flow as it moves along the intervane channel gradually decreases, and at a certain length it can become equal to zero. Thus, the relations between $\omega_{1}^{/}$and $\omega_{1}^{/ /}$can be represented by the dependence:

$$
\omega_{1}^{\prime /}=m \omega_{1}^{\prime},
$$

where $0<m<1$ is the transparency coefficient of the blades row. The value $m$ depends on the density of the blades row, which for a circular row can be represented by the ratio

$$
m=\frac{l}{t^{\prime}}
$$

where $l$ - blade chord length; $t$ - the blades spacing on the average row diameter.

Based on the transparency of the rows of the centrifugal turbo machines impellers, the absolute flow velocity deviates during their operation. Parallelograms of the velocities at the exit of the impeller with a transparent blade row with an output angle $\beta_{2}$ for all operating modes under consideration are shown in FIG. 2

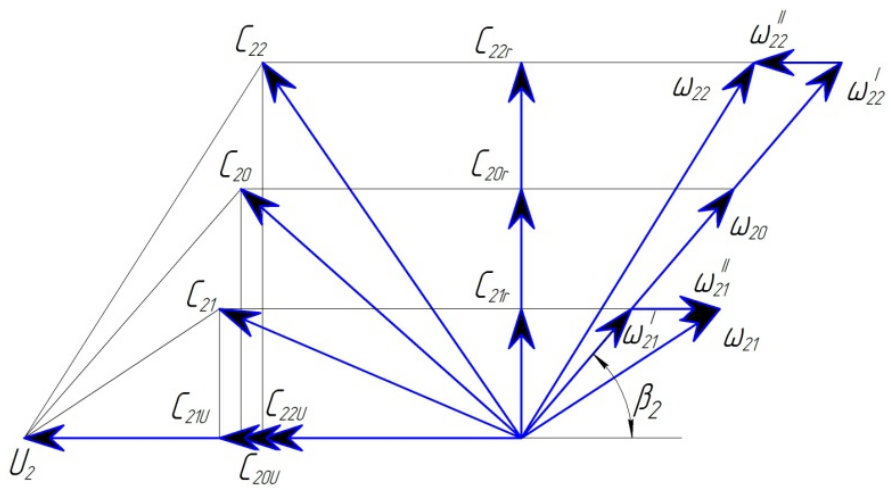

Fig. 2. Parallelograms of the average flow velocities at the outlet of the centrifugal turbo machine impeller

On the basis of the vector diagrams presented in Figures 1 and 2, we represent the values of the circumferential components of the absolute flow velocities at the input $\mathrm{c}_{1 u}$ and at the output $\mathrm{c}_{2 u}$ of the impeller:

$$
\begin{aligned}
& c_{1 u}=u_{1}-\omega_{1}^{\prime} \cos \beta_{1} \pm \omega_{1}^{\prime \prime} \\
& c_{2 u}=u_{2}-\omega_{2}^{\prime} \cos \beta_{2} \pm \omega_{2}^{\prime /}
\end{aligned}
$$

Where symbol «-» corresponds to the operating mode when $Q_{T}\left\langle Q_{T O}\right.$; «+» $Q_{T}>Q_{T O}$.

Substituting this expression in the equation of theoretical Euler's head, taking into account the relation

$\omega_{2}^{/ /}=m \omega_{1}^{/ /}$we get

$$
H_{T}=\frac{\left(u_{2}^{2}-u_{1}^{2}\right)-\left(u_{2} \omega_{2}^{\prime} \cos \beta_{2}-u_{1} \omega_{1}^{\prime} \cos \beta_{1}\right) \pm \omega_{1}^{\prime \prime}\left(u_{1}-m u_{2}\right)}{g}
$$

If there is no flow swirl at the input, when $c_{1 u}=0$, this expression reduces to the form: 


$$
\begin{aligned}
& H_{T}=\frac{u_{2}^{2}-u_{2} \omega_{2}^{\prime} \cos \beta_{2} \pm u_{2} m \omega_{1}^{/ /}}{g} \\
& \omega_{1}^{/ /}=u_{1}-\omega_{1}^{\prime} \cos \beta_{1}
\end{aligned}
$$

It's obvious that $u_{1}=u_{2}\left(\frac{D_{1}}{D_{2}}\right)$

We introduce the radial components of the absolute flow velocities at the input $c_{1 r}$ and outlet $c_{2 r}$ of the impeller based on the ratios:

$$
\begin{aligned}
& c_{1 r} \operatorname{ctg} \beta_{1}=\omega_{1}^{\prime} \cos \beta_{1} \\
& c_{2 r} \operatorname{ctg} \beta_{2}=\omega_{2}^{\prime} \cos \beta_{2}
\end{aligned}
$$

and express them through a theoretical presentation $Q_{T}$ (without leakage)

$$
\begin{gathered}
c_{1 r}=\frac{Q_{T}}{F_{1} \phi_{1}} \\
c_{2 r}=\frac{Q_{T}}{F_{2} \phi_{2}}
\end{gathered}
$$

where $\quad F_{1}=\pi D_{1} b_{1} ; F_{2}=\pi D_{2} b_{2}$ - outer and inner circumference of the impeller; $D_{1}$ and $D_{2}$ - inlet and outlet flow diameters;

$b_{1}$ and $b_{2}$ - the width of the intervane channels at the inlet to the lattice of the blades and the outlet; inlet and outlet.

$$
\phi_{1} \text { and } \phi_{2} \text { - coefficients of constraint of flow by a row of blades at the }
$$

Substituting the values obtained and expression (7) into equation (6), we obtain:

$$
H_{T}=\frac{u_{2}^{2}}{g}\left(1-m \frac{D_{1}}{D_{2}}\right)-\frac{u_{2} Q_{T}}{g F_{2} \phi_{2}}\left(\operatorname{ctg} \beta_{2}-m \frac{F_{2} \phi_{2}}{F_{1} \phi_{1}} \operatorname{ctg} \beta_{1}\right)
$$

The equation of the theoretical characteristic of a centrifugal turbo machine shows the change in the specific work done by the impeller when the fluid flows through it as a function of the feed amount $Q_{T}$

The increase in the power interchange on the impeller blades becomes possible only in three cases [9]:

1) when the flow, passing through the second impeller, becomes even more twisted in the previous direction (by changing the circumferential velocity or by changing the geometric dimensions);

2) when between the first and second impellers an intermediate one is placed - a straightening device that untwists the flow in front of the inlet to the second impeller;

3) when the impellers rotate towards each other.

At the first variant, proceeding from a technical feature of centrifugal turbo machines, an increase in the impeller diameter assumes a proportional increase in the twist degree. Changing the circumferential speed of impellers rotation will contribute to a change in the proportion between the static and dynamic components in the total pressure.

The second option is the usual pattern of a fluid flow, with the corresponding energy loss values.

With the counter rotation of the impellers, the rotation of the outer impeller is taken as positive, the circumferential speed of the inner impeller is taken into account with the opposite sign, and hence the transfer of energy without twisting this flow at the outlet and without using an intermediate guide is made possible in all operating modes. 


$$
\begin{aligned}
& H_{T}=H_{1}+H_{2}=\frac{u_{12}^{2}}{g}\left(1-m \frac{D_{11}}{D_{12}}\right)-\frac{u_{12} Q_{T 1}}{g F_{12} \phi_{12}}\left(\operatorname{ctg} \beta_{12}-m \frac{F_{12} \phi_{12}}{F_{11} \phi_{11}} \operatorname{ctg} \beta_{11}\right)+ \\
& \frac{u_{22}^{2}}{g}\left(1-m \frac{D_{21}}{D_{22}}\right)-\frac{u_{21} Q_{T 2}}{g F_{22} \phi_{22}}\left(\operatorname{ctg} \beta_{22}-m \frac{F_{22} \phi_{22}}{F_{21} \phi_{21}} \operatorname{ctg} \beta_{21}\right)
\end{aligned}
$$

The main purpose of this work is to enhance the energy efficiency of the centrifugal turbo machinery, by eliminating the fixed elements of turbo machinery, namely systems and straightening apparatus, and corresponding values of hydraulic losses. Consequently, the use of the second method is eliminated. The first and the third methods are subject to further investigation.

The centrifugal turbomachine with a coaxial arrangement of the impellers, there is a failure on their use; therefore, the flow of fluid to the outer impeller blade comes with a high value of the relative velocity.

Starting from equation (12), the value of the pressure developed by a turbomachine will be determined by the efficiency of the energy increment on the blades of the outer impeller, which in its turn depends on the operating conditions of the impellers and the values of their geometric parameters.

Geometric parameters:

- density of the blade row (number of blades);

- ratio of input and output impeller diameters (D2 / D1) and (D4 / D3);

- values of input and output angles of inclination of blades of impellers;

- width of impellers.

Mode parameters:

- rotational speed of impeller;

- the relative direction of rotation.

The number of variants of a centrifugal turbomachine with a coaxial arrangement of impellers can be an infinite number. The choice of the optimal dimensions of the flow part can only be done by comparing the required number of alternatives. However, it should be noted that the determining parameter for their selection is the condition for achieving the maximum efficiency.

The constructed theoretical characteristics (according to equation 12) for a centrifugal fan VC4-75-1.52 are shown in Fig. 3.

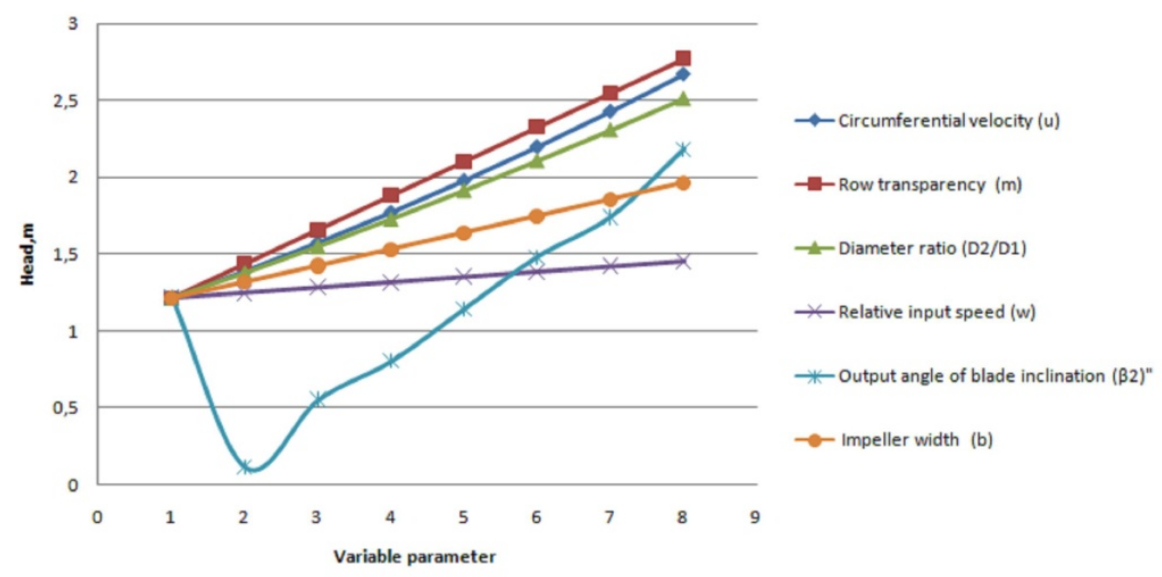

Fig. 3. Influence of geometric dimensions and flow rate when rotating impellers in one direction on the pressure developed by a turbomachine

The presented graph demonstrates the influence of geometric parameters on the pressure developed by the turbomachine, the greatest influence is made by the circumferential 
velocity, the transparency of the row (the number of blades), the ratio of the diameters (due to the geometric feature of centrifugal turbo machines and the corresponding increase in the circumferential velocity diameter). The effect of the output angle is non-linear because of the large run-up in magnitudes. The magnitude of this takeoff is dictated by the need to take into consideration all possible angles of the blades inclination.

The effect of counter rotation is shown in FIG. 4. In this case, the influence of geometric parameters is evaluated separately for two versions [10]:

$$
\begin{aligned}
& -\beta_{2}>90^{0} \\
& -\beta_{2}<90^{\circ}
\end{aligned}
$$

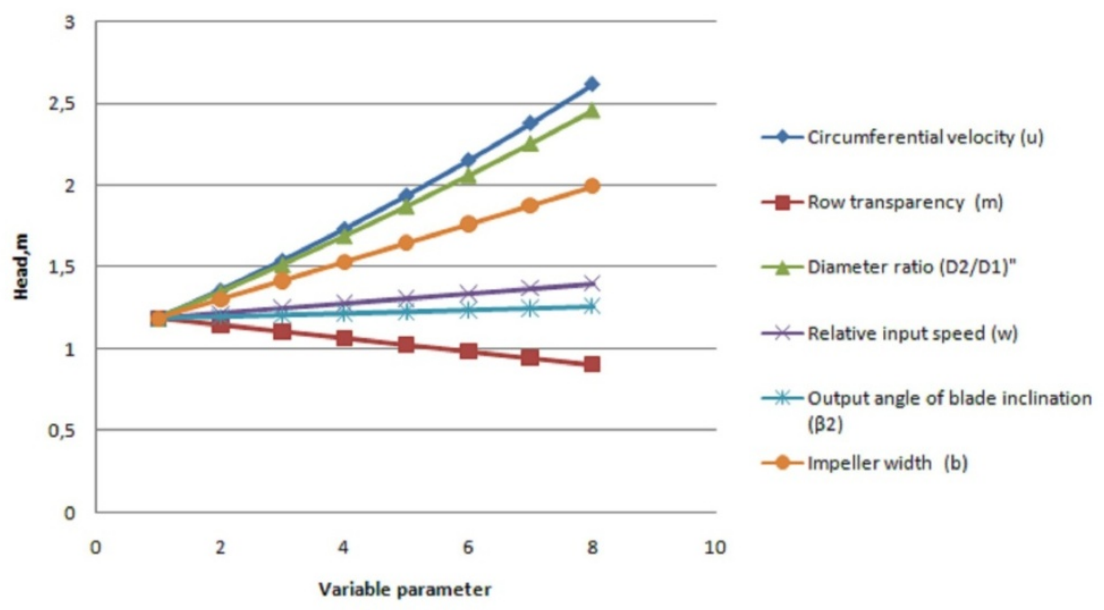

Fig. 4. Influence of geometric dimensions and flow rate when rotating impellers in opposite directions on the pressure developed by a turbomachine

\section{Conclusions}

Based on the presented graphs, it is obvious that irrespective of the operating mode, the influence of the circumferential velocity, the ratio of the input and output diameters and the width of the impeller on the magnitude of the pressure developed by the turbomachine are unchanged. While the influence of the blade rows density with the counter rotation of the impellers decreases.

To assess the power efficiency of the effect of each geometric parameter on the pressure developed by the turbomachine, it will be necessary to analyze the internal hydrodynamic losses in the impeller.

\section{References}

1. I.E. Lobanov, Bull. of NMSTU. 1 (2016)

2. V.N. Makarov, S.A. Gorbunov, T.A. Kornilova, Min. J. 6 (2013)

3. Yu.B. Galerkin, A.F. Rekstin, K.V. Soldatov et al, Compr. Eng. and pneum. 7 (2015)

4. S.G. Chernyy, D.V. Chirkov, V.N. Lapin, Numerical modelling of flows in turbomachines (Science, Novosibirsk, (2006)

5. M.A. Kol'ga, K.N. Vdovin, A.D. Kol'ga, Patent of the RF No. 77917. A centrifugal machine for transporting a fluid. - 2008121557/22, publ. 27.05.2008. 
6. S.V. Podbolotov, A.D. Kolga, Conf. Mountain Electromechanics (Perm Publishing. nat. Res. polytechnical. Univ., Perm, 2015)

7. Yu.N. Sokolov, Izvestia of Tomsk Polytechnic University. 80 (1955)

8. I.M. Zhumakhov, Pumps, fans and compressors (Uglethekhizdat, Moscow, 1958)

9. A.A. Zharkovsky, Current state and prospects for the development of hydro machinery in the 21 st century (2003)

10. S.V. Podbolotov, A.D. Kolga, Extraction, processing and application of natural stone. (2016) 